OPEN ACCESS

Edited by: William Edson Aaronson, Temple University, United States

Reviewed by:

Margo Bergman, University of Washington Tacoma, United States Jeff Bolles,

University of Mount Olive, United States

*Correspondence: Jeffrey M. Taekman jeffrey.taekman@duke.edu (jmt)

Specialty section: This article was submitted to Public Health Education and Promotion, a section of the journal

Frontiers in Public Health

Received: 28 June 2017 Accepted: 01 September 2017 Published: 26 September 2017

Citation:

Taekman JM, Foureman MF, Bulamba F, Steele M, Comstock E, Kintu A, Mauritz A and Olufolabi A (2017) A Novel Multiplayer Screen-Based Simulation Experience

for African Learners Improved Confidence in Management of Postpartum Hemorrhage.

Front. Public Health 5:248. doi: 10.3389/fpubh.2017.00248

\section{A Novel Multiplayer Screen-Based Simulation Experience for African Learners Improved Confidence in Management of Postpartum Hemorrhage}

\author{
Jeffrey M. Taekman ${ }^{1,2,3 *}$, Megan F. Foureman², Fred Bulamba4 ${ }^{4}$, Michael Steele ${ }^{3}$, \\ Emily Comstock ${ }^{5}$, Andrew Kintu ${ }^{4}$, Amy Mauritz ${ }^{1,2}$ and Adeyemi Olufolabi ${ }^{1,2}$
}

\begin{abstract}
${ }^{1}$ Department of Anesthesiology, Duke University School of Medicine, Durham, NC, United States, ${ }^{2}$ Duke University Medical Center, Durham, NC, United States, ${ }^{3}$ Duke University School of Medicine, Durham, NC, United States, ${ }^{4}$ Department of Anesthesia, Makerere University College of Health Sciences, Kampala, Uganda, ${ }^{5}$ Duke University School of Nursing, Durham, NC, United States
\end{abstract}

Introduction: Postpartum hemorrhage (PPH) remains a global challenge, affecting thirteen million women each year. In addition, PPH is a leading cause of maternal mortality in Asia and Africa. In the U.S.A., care of critically ill patients is often practiced using mannequin-based simulation. Mannequin-based simulation presents challenges in global health, particularly in low- or middle-income countries. We developed a novel multiplayer screen-based simulation in a virtual world enabling the practice of team coordination with $\mathrm{PPH}$. We used this simulation with learners in Mulago, Uganda. We hypothesized that a multiplayer screen-based simulation experience would increase learner confidence in their ability to manage $\mathrm{PPH}$.

Methods: The study design was a simple pre- and a post-intervention survey. Fortyeight interprofessional subjects participated in one of nine 1-h simulation sessions using the PPH software. A fifteen-question self-assessment administered before and after the intervention was designed to probe the areas of learning as defined by Bloom and Krathwohl: affective, cognitive, and psychomotor.

Results: Combined confidence scores increased significantly overall following the simulation experience and individually in each of the three categories of Bloom's Taxonomy: affective, cognitive, and psychomotor.

Conclusion: We provide preliminary evidence that multiplayer screen-based simulation represents a scalable, distributable form of learning that may be used effectively in global health education and training. Interestingly, despite our intervention being screen-based, our subjects showed improved confidence in their ability to perform psychomotor tasks. Although there is precedent for mental rehearsal improving performance, further research is needed to understand this finding.

Keywords: distance education, educational technology, medical education, serious games, simulation 


\section{INTRODUCTION}

Despite improvements in the rate of maternal mortality worldwide, more than 250,000 women die each year during the perinatal period (1), a good number from postpartum hemorrhage $(\mathrm{PPH})$. PPH remains a global challenge, affecting thirteen million women each year (2) and is a leading cause of maternal mortality in Asia and Africa (3).

Treating PPH requires two critical steps: recognition of the event and an appropriate clinical and timely management of bleeding (3). In low- and middle-income countries (LMIC), failing either of these steps often leads to maternal death. A great deal of PPH management involves teamwork and communication. Thus, teamwork and communication training is a critical component in the management of any life-threatening event.

Communication failures are responsible for approximately two-thirds of iatrogenic harm in healthcare (4). Over the last decade, in the United States (U.S.), teamwork and communication has been recognized as a critical component of clinical management, largely in response to the Institute of Medicine's (IOM) To Err is Human (5). However, learning to function as a team takes practice (4). In response to the call to action by the IOM, the U.S. Department of Defense and the Agency for Healthcare Research and Quality developed TeamSTEPPS, "a flexible, evidence-based toolkit to improve patient safety through enhanced communication and other teamwork skills." (6) The TeamSTEPPS curriculum leverages two decades of experience with teamwork and communication in high-risk industries. These materials are freely distributed over the internet and can be customized to any healthcare setting.

Over the past two decades, there has been explosive growth in the use of mannequin-based simulation in healthcare education and training (7). Teamwork and communication is often taught using mannequin-based simulation. High-fidelity, mannequinbased simulation recreates an environment where learners manage realistic patients in context (8). Students practice their profession in scripted scenarios designed to challenge clinical decision-making, critical thinking, and teamwork. What makes simulation so effective, in all of its forms, is it allows learners to practice in the context of how the learning will be applied, a construct called situated cognition (9). Simulation has many advantages over lecture-based education, including interactivity, reproducibility, and the ability to allow learners to practice in teams and in context (8).

Simulations, both high and low fidelity, are effective training methods for obstetric emergencies (10). Although mannequinbased simulation is an effective high-fidelity training method, it has limitations in global health. Mannequin-based simulation, when used correctly, requires expensive equipment, a complex video and computer infrastructure, and dedicated, specially trained personnel. Other major limitations include the requirement for individuals to co-locate and the difficulty scaling training to a large learner population (8). Moving simulation equipment internationally to LMICs adds significantly to the logistical complexity and expense of training.

More recently, largely due to the increase in power of computers, increasing speed of the internet, and capabilities of software, screen-based simulation has become more common in healthcare education $(8,11)$. Some screen-based simulation leverages commercial gaming technology. Screen-based simulation offers many advantages over mannequin-based simulation, especially for global health. These advantages include the ability to run software on relatively inexpensive computers, the ability to easily distribute and scale, and may be accessed regardless of the learner's location (8). In addition, because screen-based simulation takes place on a computer over a network, every decision and action taken by the learner becomes a data point, enabling analysis through learning analytics (big data for education) (11).

In collaboration with a local government contractor (Applied Research Associates, Cary, NC, USA), we developed a multiplayer screen-based simulation that enables the practice of teamwork and communication (using the TeamSTEPPS) in the setting of $\mathrm{PPH}$. The software is one of several packages under the Immersive Learning Environments @ Duke (ILE@D) Umbrella (http:// lr.simcenter.duke.edu/) - The PPH software enables interprofessional learners, regardless of their location in the world, to share a virtual birthing room and care for a virtual patient (Figure 1). Simulation with the software is similar to that of mannequinbased simulation in that learners participate in the virtual care of a patient, then reflect on their actions during a formal debrief. These debriefs may be held locally or may leverage digital technology (e.g., Skype) to conduct the discussion at a distance.

We undertook a proof-of-concept training program using our software for PPH education in Uganda. We hypothesized that our multiplayer screen-based simulation would be effective in increasing learner confidence in the management of $\mathrm{PPH}$.

\section{MATERIALS AND METHODS}

\section{Simulation}

In Spring 2015, we conducted nine 1-hour sessions over a 5-day period. Due to the educational nature of this study, it was not registered in a clinical trial registry. Following IRB approval and written informed consent, 48 subjects were recruited through flyers placed at Mulago Hospital/Makerere University in Mulago, Uganda. Two to ten interprofessional participants were present for each session. Learners were trainees or professionals from: Obstetrics, Midwifery, and Anesthesiology.

Our software, built on the Epic Games platform (Cary, NC, USA). Epic is the technology behind many commercial video games. Epic runs on inexpensive, easily accessible computers with internet connections. In our case, the Epic technology was repurposed to develop a healthcare learning activity. The simulation was customized to reflect available resources in Uganda (e.g., whole blood only instead of multiple blood fractions). Learners participated in the simulation from individual computers, controlling the actions of their character using a computer mouse. Voice over IP (VOIP) enables audio communication over the internet. Participants wore VOIP headsets with microphones in order to facilitate teamwork and communication.

After a brief introduction to the project, each learner practiced moving their avatar in the virtual environment, interacting 


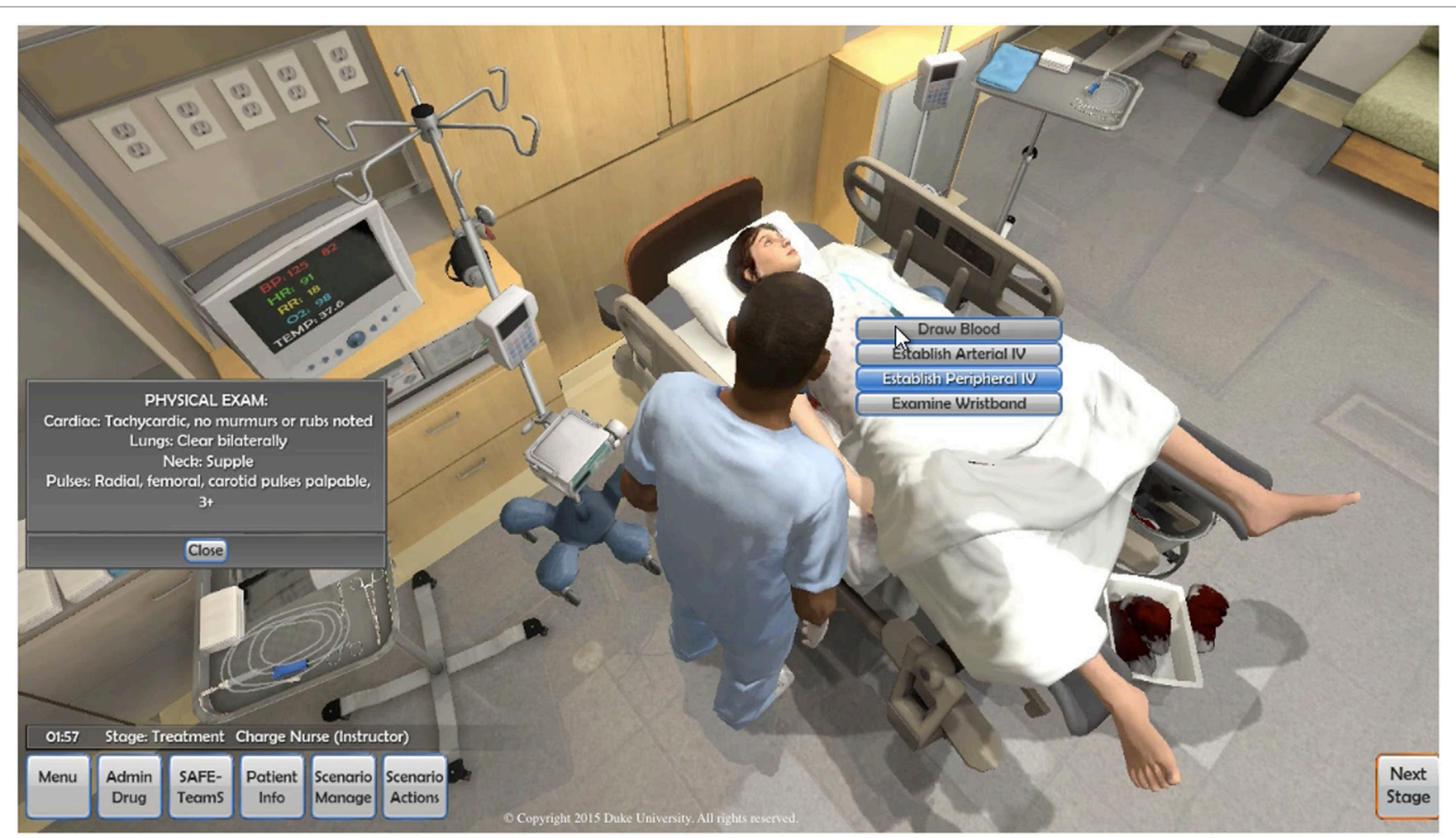

FIGURE 1 | Screen-shot of 3DiTeams - postpartum hemorrhage-multiplayer screen-based simulation. Each character is controlled by a unique individual using a computer, mouse, and voice-over-IP headset.

with equipment, the patient, and other learners. In addition, each participant practiced administering drugs as well as verifying and administering blood products. Following this introductory period, the simulation was re-initiated for the actual learning experience.

Learners participated in the same, instructor lead simulation experience focused on $\mathrm{PPH}$. The simulation experience consisted of a 30-min screen-based facilitator-lead simulation followed by a 30-min debrief with faculty both in Uganda and in the United States. The participants communicated with distant faculty using VOIP headsets.

The sessions in Uganda were led by a trained simulation facilitator from the Duke University Human Simulation and Patient Safety Center. Team members in the US were able to watch, listen, and participate in the simulation in real time. For the debrief, the facilitator in Uganda was joined in the debrief by team members in the US via Skype (Microsoft, Redmond, WA, USA).

\section{Study}

The study design was a pre- and a post-intervention survey. In addition to demographic information, questions to probe the learners' familiarity with computers and computer games were included.

A fifteen-question self-assessment (Appendix A in Supplementary Material) was designed to probe the three areas of learning as defined by Bloom and Krathwohl: psychomotor, cognitive, and affective (12). The questionnaire consisted of four psychomotor questions, six cognitive questions, and five affective questions targeted at management of $\mathrm{PPH}$. The questionnaire used a scale from 0 (not confident) to 10 (very confident). Each participant completed the questionnaire before and after their simulation experience.

The confidence questions (Appendix A in Supplementary Material) were meant to reflect representative tasks of management of PPH. Affective tasks included such items as comfort with the obstetric environment, and the ability to communicate effectively with team members. Cognitive tasks included recognizing and treating $\mathrm{PPH}$, timing and administration of drugs, and correct responses to alterations in patient condition. Psychomotor tasks included performance of fundal massage, placement of intravenous lines, and administration of medications via the correct route.

In addition to post-intervention confidence questions, the learners answered a series of questions to gage their immersion in the training, the time spent in the simulation, and their thoughts about screen-based simulation.

Data were collected on paper by trainers before and immediately after the intervention. Confidence data, pre- and postsimulation, were compared using a paired $t$-test comparison. Comparison data are reported as mean $\pm \mathrm{SD}$.

\section{RESULTS}

Learners were $60 \%$ male and $40 \%$ female (See Table 1). Age range was 21-55 years with an average age of 31.2 years. 
TABLE 1 | General demographics of subjects.

\begin{tabular}{lc}
\hline Age range (years) & $21-55$ \\
Average age (years) & 31.2 \\
Median age (years) & 29 \\
Gender & \\
Male & $60 \%$ \\
Female & $40 \%$ \\
Obstetric experience & $96 \%$ \\
Mannequin sim experience & $45 \%$ \\
Screen-Based sim experience & $15 \%$ \\
Profession & \\
Obstetrician & $10 \%$ \\
Midwife & $15 \%$ \\
Anesthetic Officer & $48 \%$ \\
Anesthesiologist & $27 \%$ \\
\hline
\end{tabular}

Learners' professions were 10\% Obstetrics, 15\% Midwifery, 48\% Anesthetic Officers, and 27\% Anesthesiologists. Midwives, not nurses, participate in live births at Makerere University Hospital.

Ninety-six percent of learners had at least some experience on the obstetrics ward. Fifty-six percent of learners had little or no experience working with computers. Although $46 \%$ of learners had participated in a mannequin-based simulation, only $15 \%$ had experienced a computer-based simulation. Despite the limited exposure prior to our intervention, $87 \%$ believed screen-based simulation would be an effective way to learn the skills of caring for a woman with PPH. Eighty-two percent of learners played 2 hours or less a week of video games. Ninety-six percent of the participants had never played First Person Shooter games (the software platform the simulation is built upon).

Self-reported confidence scores increased significantly overall following the simulation experience ( $p r e=7.83 \pm 1.55$, post $=8.95 \pm 1.42, p<0.001$ ) (Figure 2A and Table 2) and individually in each of the three categories of Bloom's Taxonomy: affective (pre $=7.70 \pm 1.75$, post $=9.00 \pm 1.46, p<0.001$ ), psychomotor (pre $=8.31 \pm 1.71$, post $=9.11 \pm 1.51, p<0.001)$, and cognitive (pre $=7.46 \pm 1.89$, post $=8.73 \pm 1.51, p<0.001)$ (Figure 2B and Table 2).

Following the simulation and debrief, $100 \%$ of learners felt screen-based simulation was an effective way to learn to care for a woman suffering from PPH. Ninety-eight percent of participants would recommend the simulation as a way to learn about PPH.

Following the simulation, learners were asked various questions about the software. Ninety-six percent of learners agreed or strongly agreed with the statement, "It is important to have access 24 hours a day, 7 days a week, 365 days a year." Eighty-four percent agreed or strongly agreed with the statement, "I would like to participate in the simulation on my own device (rather than being required to use a dedicated computer)." Learners were mixed in their interest in having collaborators in the US-23\% disagreed or strongly disagreed this was necessary, 52\% agreed or strongly agreed, and $23 \%$ were neutral.

Learners valued the multiplayer aspect of the simulation. When asked if they would like to learn independently, without the need for other learners, $63 \%$ disagreed or strongly disagreed, $20 \%$ agreed, and $17 \%$ were neutral. When asked if they would like to continue to learn in an environment with other human team players, $98 \%$ agreed and $2 \%$ were neutral.
The learners valued feedback, both in-game and by a live facilitator. When asked if it is important to have immediate, in-game feedback, $91 \%$ agreed, $2 \%$ disagreed, and $7 \%$ were neutral. When asked if it is important to have feedback in a debrief with a live facilitator, $94 \%$ agreed or strongly agreed, $2 \%$ disagreed, and $4 \%$ were neutral.

\section{DISCUSSION}

This is the first description of a multiplayer screen-based simulation/virtual environment used in global health. Our study with learners in Uganda, using our novel software as the centerpiece of a simulation experience, demonstrated an increase in participants' confidence in the management of $\mathrm{PPH}$. In addition, there was wide consensus that screen-based simulation was an effective way to practice the teamwork needed for proper management of $\mathrm{PPH}$. Confidence improved in all three domains of Bloom's Taxonomy: affective, cognitive, and psychomotor. Since learners do not perform any physical procedure in screen-based simulation, a counterintuitive finding was a gain in psychomotor confidence following participation in the session.

Simulation provides an opportunity for mental rehearsal and reflection. Although more research is needed to understand how increased confidence translates into real-world performance, there is precedent for mental rehearsal improving performance in sports (13) and medicine (14). Mental reflection is also a key component in the development of expertise (15). The initial implication is that screen-based simulation, despite lacking a psychomotor component, may be a viable substitute for at least a subset of mannequin-based simulation activities. It must be pointed out that the primary focus of our simulation was teamwork and communication and only included simple psychomotor tasks. Screen-based simulation might not have been as effective in changing psychomotor confidence with more complex psychomotor tasks such as a surgical procedure. More research is needed to understand the impact of screenbased simulation on the acquisition, performance, and mastery of affective, cognitive, and psychomotor skills in the real world, both simple and complex.

Screen-based simulation is infinitely more scalable and portable than mannequin-based simulation $(8,11)$ and offers many other advantages and opportunities for global health. These advantages include convenience for the learners and facilitators, ubiquity of equipment to run software, and overall lower cost. Because the software runs on readily available computers, screen-based simulation enables new opportunities for global health, including just-in-time education from a distant partner (USA) to anywhere (Figures $\mathbf{3 A}, \mathbf{B}$ ) or anywhere to anywhere (Figure $3 \mathrm{C}$ ). Configurations such as those noted in Figure $3 \mathrm{C}$, since they require no more than a computer and VOIP headset, may be scaled to a global audience relatively quickly. Furthermore, the interconnectedness of the internet can form a network of educators and learners that may link with one another repeatedly, regardless of location. The interconnectedness of this learning network enables a wide array of new learning activities and data opportunities. Marrying the data 
A

$\mathrm{p}<0.0001$

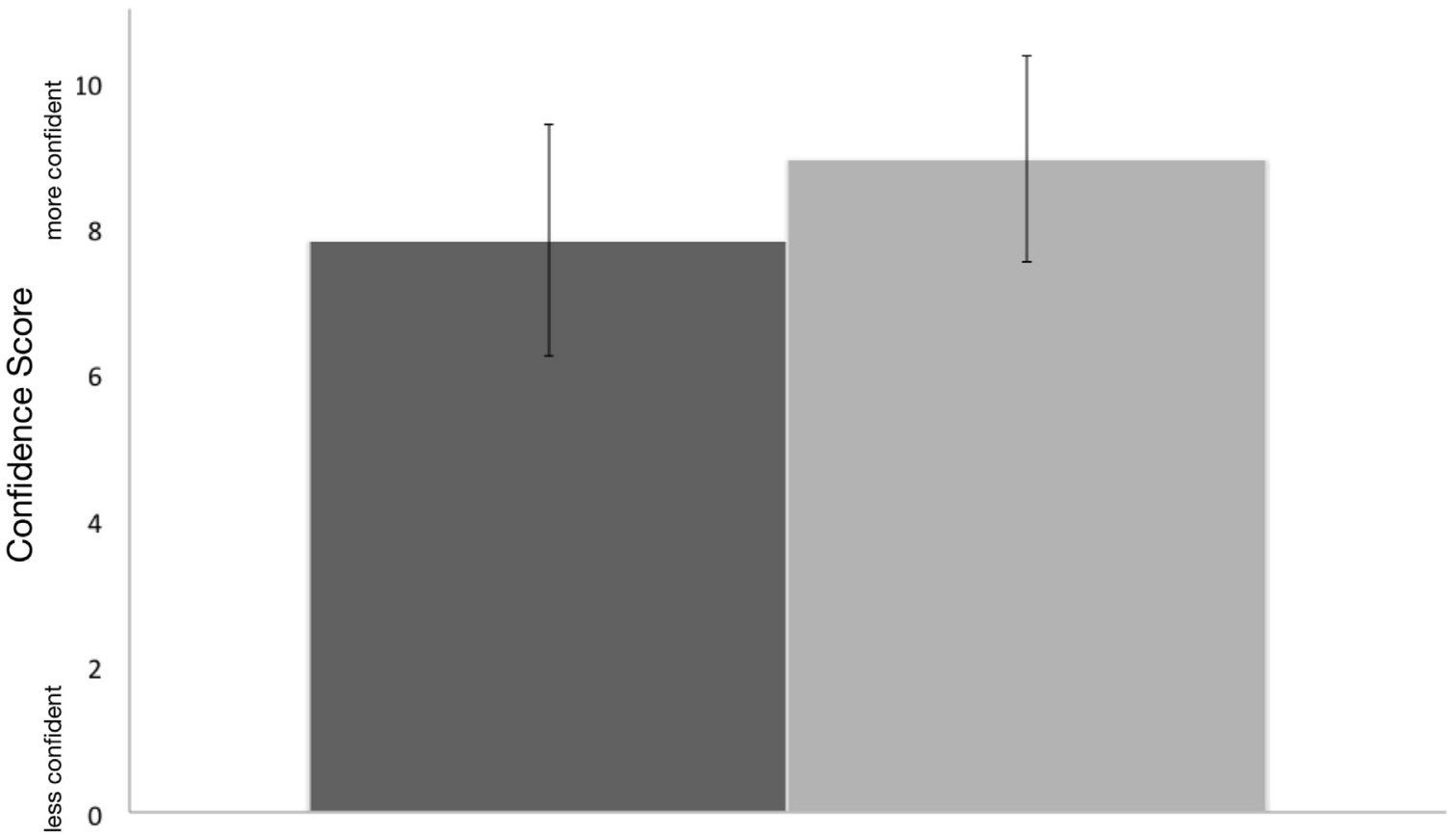

Pre Sim

Post Sim

B

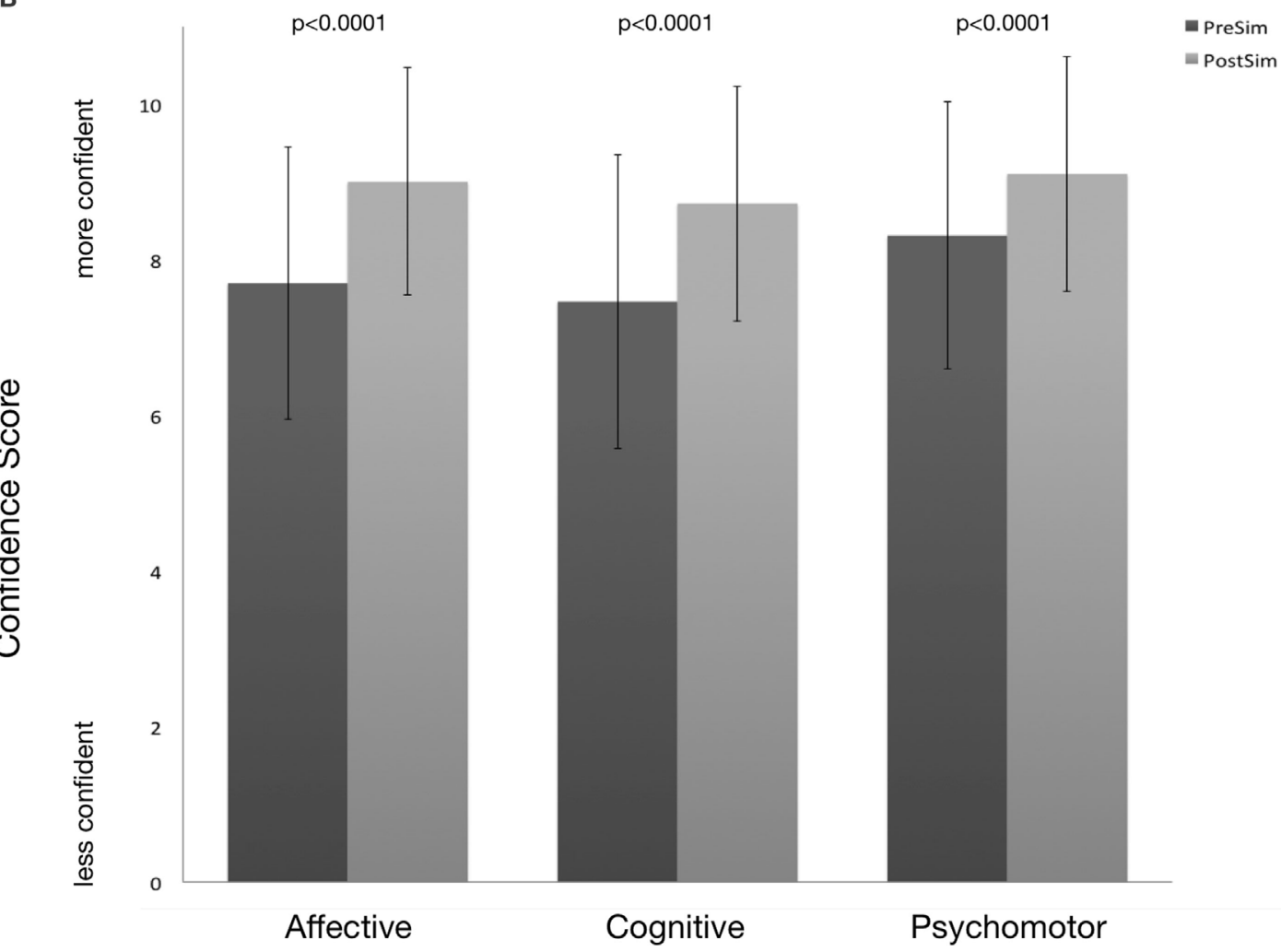

FIGURE 2 | (A,B) Pre- and post-learner confidence in management of postpartum hemorrhage (value \pm S.D.) Questions were categorized into one of three domains of Bloom's Taxonomy. Pre and post survey consisted of four psychomotor questions, six cognitive questions, and five affective questions. Following the screenbased simulation experience, significant gains in confidence were seen in each of the domains (B) as well as the combined overall confidence score (A). 
generated from our simulation with other data sources could usher in a brand new area of investigation for Global Health: Learning Analytics.

It should be noted that although only a portion of our learners had experience with gaming, they universally found the screen-based simulation useful. Although not reflected in our data, one of the common comments was how individual learners enjoyed the interprofessional nature of the exercise. Many cited interprofessional education as the most valuable part of the entire experience. Mannequin-based simulation is known to be an effective method of training teams. It appears,

TABLE 2 | Pre and post learner confidence in management of postpartum hemorrhage (value $\pm \mathrm{SD}$ ).

\begin{tabular}{lccc}
\hline Variable & Pre & Post & $\boldsymbol{p}$ \\
\hline Affective & $7.70 \pm 1.75$ & $9.00 \pm 1.46$ & $<0.0001$ \\
Cognitive & $7.46 \pm 1.89$ & $8.73 \pm 1.51$ & $<0.0001$ \\
Psychomotor & $8.31 \pm 1.72$ & $9.11 \pm 1.51$ & $<0.0001$ \\
Ave. combined & $7.83 \pm 1.58$ & $8.95 \pm 1.42$ & $<0.0001$
\end{tabular}

Questions were categorized into one of three domains of Bloom's Taxonomy. Pre and post survey consisted of four psychomotor questions, six cognitive questions, and five affective questions. Following the screen-based simulation experience, significant gains in confidence were seen in each of the domains as well as the combined overall confidence score. from our results, that screen-based simulation may be equally effective.

Our study has several limitations. Participants self-selected and, thus, may not be a truly representative sample of providers in Uganda. Furthermore, one must consider the simulation experience may not be directly responsible for the gains in learner confidence. Instead, learner improvements may have been a result of prolonged reflection on their performance. Even if this is the case, the PPH software offers a viable alternative to the way most learners in LMICs gain experience: with live (and sometimes critically ill) patients. Our PPH software set the stage for situated cognition (9)-placing the learner in the clinical environment-without risk to patients.

The original software was designed for learners in a highly resourced medical center environment. The virtual environment setting - a state-of-the-art birthing room-would be unfamiliar to learners in LMICs. Despite these incongruities in appearance, the software was effective in changing learner confidence although it is unknown what the impact of these incongruences might be.

Although our findings are promising, our results may not be generalized to other LMICs. Each country (and sometimes even hospital) has its own unique culture, hierarchy, and patient-care challenges. The challenges seen in other locations may be less

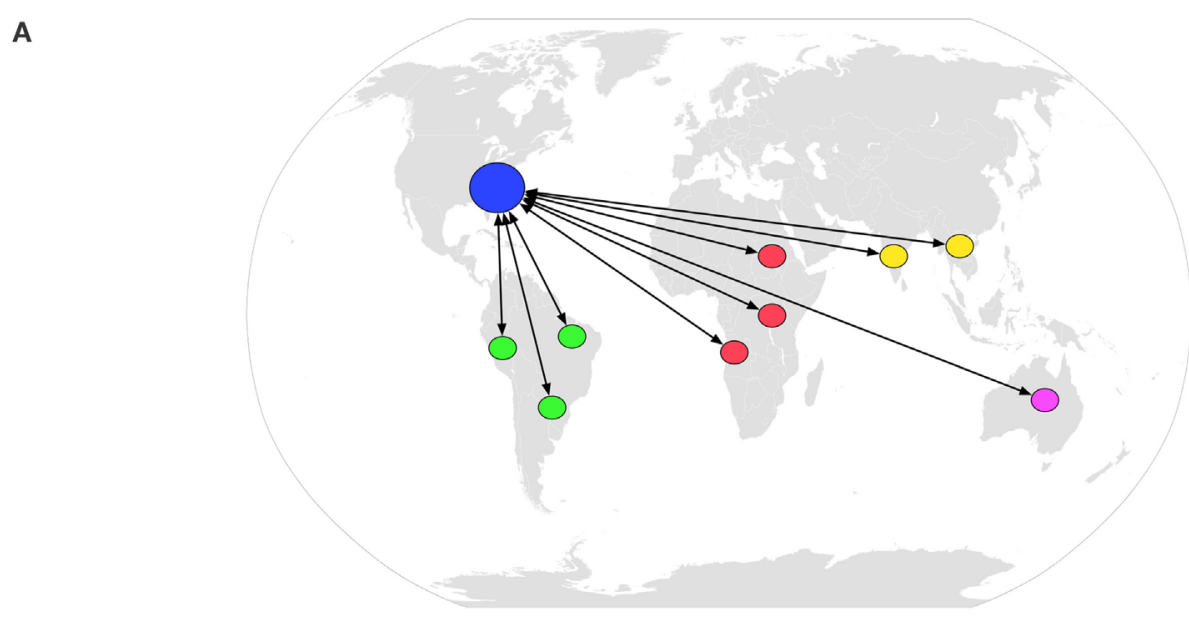

B

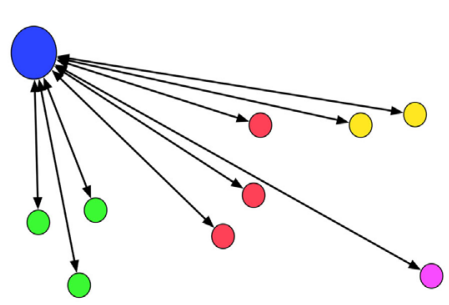

C

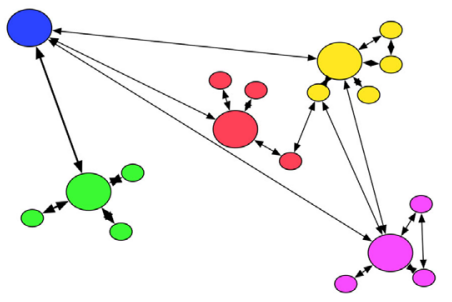

FIGURE 3 | Visual representation of new opportunities enabled by multiplayer screen-based simulation. Because the software runs on readily available computers, screen-based simulation enables new opportunities for global health, including just-in-time education from the USA to anywhere (A,B) or anywhere to anywhere (C). Configurations such as those noted in Figure $\mathbf{3 C}$, since they require no more than a computer and Voice-over-IP headset, may be scaled to a global audience relatively quickly. 
amenable to change by our software. Further research is needed to understand how our findings generalize to other settings.

Self-reported outcomes have their own limitations. Ultimately educators and administrators want proof that screen-basedsimulation impacts patient outcomes. Much as with the case of mannequin-based simulation, definitive proof with patient outcomes may never be possible, given the relatively low frequency and unexpected nature of critical events (16).

\section{CONCLUSION}

In this report, we provide preliminary evidence that multiplayer screen-based simulation may be used effectively in global health education/clinical training. We foresee screen-based simulation ushering in a new generation of opportunities in global health education.

\section{ETHICS STATEMENT}

This study was approved by the Duke University Medical Center IRB Department. All subjects were properly consented before participating in the study. Please see contact information below for Duke University IRB Office. Duke University IRB Office Hock Plaza, Suite 4052424 Erwin Road Campus Box \#2712 Durham, NC 27705 (919) 668-5111.

\section{REFERENCES}

1. Bustreo F, Say L, Koblinsky M, Pullum TW, Temmerman M, PablosMéndez A. Ending preventable maternal deaths: the time is now. Lancet Glob Health (2013) 1:e176-7. doi:10.1016/S2214-109X(13)70059-7

2. Evans CL, Johnson P, Bazant E, Bhatnagar N, Zgambo J, Khamis AR. Competency-based training "Helping Mothers Survive: bleeding after Birth" for providers from central and remote facilities in three countries. Int J Gynaecol Obstet (2014) 126:286-90. doi:10.1016/j.ijgo.2014.02.021

3. Perosky J, Richter R, Rybak O, Gans-Larty F, Mensah MA, Danquah A, et al. A low-cost simulator for learning to manage postpartum hemorrhage in rural Africa. Simul Healthc (2011) 6:42-7. doi:10.1097/SIH.0b013e3181 ebbcfd

4. Leonard M, Graham S, Bonacum D. The human factor: the critical importance of effective teamwork and communication in providing safe care. Qual Saf Health Care (2004) 13(Suppl 1):i85-90. doi:10.1136/qshc. 2004.010033

5. Institute of Medicine (US) Committee on Quality of Health Care in America, Kohn LT, Corrigan JM, Donaldson MS. To Err is Human: Building a Safer Health System. Washington, DC: National Academies Press (US) (2000).

6. Clancy CM, Tornberg DN. TeamSTEPPS: assuring optimal teamwork in clinical settings. Am J Med Qual (2007) 22:214-7. doi:10.1177/1062860607300616

7. Ross AJ, Kodate N, Anderson JE, Thomas L, Jaye P. Review of simulation studies in anaesthesia journals, 2001-2010: mapping and content analysis. Br J Anaesth (2012) 109:99-109. doi:10.1093/bja/aes 184

8. Taekman J, Shelley K. Virtual environments in healthcare: immersion, disruption, and flow. Int Anesthesiol Clin (2010) 48:101-21. doi:10.1097/ AIA.0b013e3181eace73

9. Gee JP. What video games have to teach us about learning and literacy. Comput Entertain (2003) 1:20-20. doi:10.1145/950566.950595

10. Calvert KL, Mcgurgan PM, Debenham EM, Gratwick FJ, Maouris P. Emergency obstetric simulation training: how do we know where we are going, if

\section{AUTHOR CONTRIBUTIONS}

JT: designed the study, conducted the study, analyzed and interpreted the data, and prepared the manuscript. MF: helped design the study, conducted the study, collected the data, confirmed analysis, and prepared the manuscript. FB, MS, EC, and AK: conducted the study, collected data, and prepared the manuscript. AM: conducted the study and prepared the manuscript. AO: conducted the study, collected data, and prepared the manuscript.

\section{FUNDING}

This project was made possible through the generous funding of The Social Entrepreneurship Accelerator at Duke and The Duke Endowment. The Social Entrepreneurship Accelerator at Duke (SEAD) is funded by the United States Agency for International Development (USAID) under cooperative agreement number AID-OAA-A-13-00004. Immersive Learning Environments @ Duke (ILE@D) is funded by a grant from The Duke Endowment.

\section{SUPPLEMENTARY MATERIAL}

The Supplementary Material for this article can be found online at http://journal.frontiersin.org/article/10.3389/fpubh.2017.00248/ full\#supplementary-material.

we don't know where we have been? Aust NZ J Obstet Gynaecol (2013) 53:509-16. doi:10.1111/ajo.12120

11. Owens R, Taekman J. Virtual reality, haptic simulators, and virtual environments. In: Levine AI, DeMaria S Jr., Schwartz AD, Sim AJ, editors. The Comprehensive Textbook of Healthcare Simulation. New York: Springer (2013). p. 233-53.

12. Bloom B, Krathwohl D. Taxonomy of Educational Objectives: The Classification of Educational Goals. New York: Longmans, Green (1956).

13. Saimpont A, Lafleur MF, Malouin F, Richards CL, Doyon J, Jackson PL. The comparison between motor imagery and verbal rehearsal on the learning of sequential movements. Front Hum Neurosci (2013) 7:773. doi:10.3389/ fnhum.2013.00773

14. Jackson PL, Lafleur MF, Malouin F, Richards C, Doyon J. Potential role of mental practice using motor imagery in neurologic rehabilitation. Arch Phys Med Rehabil (2001) 82:1133-41. doi:10.1053/apmr.2001.24286

15. Ericsson AK, Ericsson AK, Nandagopal K, Nandagopal K, Roring RW, Roring RW. Toward a science of exceptional achievement: attaining superior performance through deliberate practice. Ann N Y Acad Sci (2007) 1172: 199-217. doi:10.1196/annals.1393.001

16. Gaba DM. The future vision of simulation in health care. Qual Saf Health Care (2004) 13(Suppl 1):i2-10. doi:10.1136/qshc.2004.009878

Conflict of Interest Statement: JT has proprietary interest in the simulation platform should it be commercialized.

Copyright (c) 2017 Taekman, Foureman, Bulamba, Steele, Comstock, Kintu, Mauritz and Olufolabi. This is an open-access article distributed under the terms of the Creative Commons Attribution License (CC BY). The use, distribution or reproduction in other forums is permitted, provided the original author(s) or licensor are credited and that the original publication in this journal is cited, in accordance with accepted academic practice. No use, distribution or reproduction is permitted which does not comply with these terms. 